\title{
ON A CLASS OF LINEAR-STATE DIFFERENTIAL GAMES WITH SUBGAME INDIVIDUALLY RATIONAL AND TIME CONSISTENT BARGAINING SOLUTIONS
}

\author{
Simon Hoof \\ Paderborn University, Germany \\ simon.hoof@upb. de
}

\begin{abstract}
We consider $n$-person pure bargaining games in which the space of feasible payoffs is constructed via a normal form differential game. At the beginning of the game the agents bargain over strategies to be played over an infinite time horizon. An initial cooperative solution (a strategy tuple) is called subgame individually rational (SIR) if it remains individually rational throughout the entire game and time consistent (TC) if renegotiating it at a later time instant yields the original solution. For a class of linear-state differential games we show that any solution which is individually rational at the beginning of the game satisfies SIR and TC if the space of admissible cooperative strategies is restricted to constants. We discuss an application from environmental economics.
\end{abstract}

Keywords: Differential games, bargaining solutions, time consistency. JEL Classification Numbers: C61, C71, C78.

\section{INTRODUCTION}

uppose there are two countries that pollute the environment by emitting $\checkmark$ pollutants and further assume that there is a production technology that

I am indebted to Claus-Jochen Haake, three anonymous referees, and participants of the 9th Murat Sertel Workshop for valuable comments. This work was partially supported by the German Research Foundation (DFG) within the Collaborative Research Center "On-The-Fly Computing" (SFB 901) under the project number 160364472-SFB901/3.

Copyright (C) Simon Hoof / 5(1), 2020, 79-98. 
maps pollutant emissions into output and output is increasing in emissions. Hence, each country has an incentive to pollute the environment. The total pollution stock, however, is assumed to be a common bad and payoffs are thus decreasing in the total stock of pollution. Total pollution further comes with a stock externality. If one neighboring country increases the rate of emissions, then it harms the other one, because the pollution stock increases. Clearly, there are some underlying dynamics in the sense that current emissions increase the total stock of pollution over time. The problem at hand can be modeled as a differential game in which the countries are agents, emission rates are the respective action variable of each country and the pollution stock is the state variable (E. J. Dockner \& Van Long, 1993). Considering the noncooperative equilibrium as a solution of the game, it turns out that it is not efficient, because both countries are overemitting with respect to the social optimum and thus both countries could gain by coordinating their actions. The two countries face a bargaining situation in the sense of Nash (1950b, p. 155):

"A TWO-PERSON bargaining situation involves two individuals who have the opportunity to collaborate for mutual benefit in more than one way."

Liu (1973) linked cooperative bargaining games to differential games. He studied a class of differential games in normal form in which strategies are assumed to be determined by the Nash bargaining solution. Let us assume that the countries determine their current emission rate via feedback strategies, i.e., current emissions are a function of the current pollution stock. At the beginning of the dynamic game the countries bargain over the strategies to be played during the game, while the noncooperative equilibrium payoff serves as the disagreement point. They would come to an agreement if it was overall individually rational, i.e., the payoffs under cooperation exceed the noncooperative equilibrium payoffs at the beginning of the game. A bargaining solution thus determines the cooperative strategies to be played during the game. Haurie (1976) pointd out two potential reasons for the breakdown of an initial agreement:

(i) If there is an agent whose current noncooperative outside option payoff dominates the current cooperative payoff, then this agent should abandon the agreement and play noncooperatively in the remaining game.

(ii) If the agents are about to renegotiate the initial agreement at a later time instant, it is not granted that they agree on the original solution. 
The first property is called subgame individually rationality (SIR) and the second one time consistency (TC). In the literature on cooperation in differential games it is usually assumed that the agents jointly maximize the weighted sum of payoffs. The resulting strategies are the cooperative strategies to be played during the entire game and they satisfy the TC property by construction. Cooperation is assumed to last if the gains of cooperation exceed the noncooperative equilibrium payoff for each agent and at each time instant. One further distinguishes between games with transferable (Petrosyan \& Zaccour, 2018) and nontransferable utility (Yeung \& Petrosyan, 2018). If payoffs are transferable the designer tries to construct a SIR payoff distribution procedure. If payoffs are nontransferable, then the designer tries to adjust the weights in the joint maximization program such that the resulting payoffs are SIR.

The present approach deviates from the standard approach in the sense that the cooperative strategies are not determined by a joint payoff maximization program, but via a bargaining solution. The goal of the paper is to characterize a variety of linear-state infinite horizon differential games ${ }^{1}$ and show how to construct bargaining solutions that satisfy $(i)$ and $(i i)$. The advantage of linear-state games is their analytical tractability and the coincidence of open loop and feedback equilibria (E. J. Dockner et al., 2000, Ch. 7.2).

Taking the noncooperative equilibrium payoffs as given (disagreement point), we induce a cooperative $n$-person pure bargaining game by considering all strategies that payoff dominate the disagreement point. It is shown that SIR and TC bargaining solutions exist if we restrict the set of admissible cooperative strategies to constants. At a first glance it seems that the restriction of the strategy space to constants is rather limiting as we neglect the case in which strategies are time and state dependent functions. We will show, however, that the class of games under consideration exhibits a subgame perfect Nash equilibrium in constant actions. That is, even though we allow for strategies that depend on the current state and thus adjust for the evolution of the state over time, it is an equilibrium strategy to fix a constant action during the entire game.

The rest of this article is organized as follows. Section 2 discusses the relation between bargaining and differential games. Section 3 examines disagreement points. Section 4 focuses on linear-state games. Section 5

1 The same class of games is considered in Jørgensen et al. (2003). They are also concerned with subgame individually rational solutions, but the cooperative strategies are determined by maximizing the joint sum of payoffs. 
presents some applications. Section 6 concludes.

\section{LINKING BARGAINING AND DIFFERENTIAL GAMES}

Let $N=\{1,2, \ldots, n\}$ denote the set of agents. A bargaining problem $(S, \boldsymbol{d})$ is described by a set $S \subseteq \mathbb{R}^{n}$ of feasible payoffs and a given disagreement point $\boldsymbol{d} \in S$. Let $\mathcal{B}$ denote the set of all bargaining problems. A bargaining solution is a mapping $\phi: \mathcal{B} \rightarrow \mathbb{R}^{n}$ that assigns to each problem $(S, \boldsymbol{d}) \in \mathcal{B}$ a unique point in the set of feasible payoffs $\phi(S, \boldsymbol{d}) \in S$. In the present paper the set of feasible payoffs is constructed via a differential game. Therefore we first introduce the differential game terminology and then show how a differential game can be used to induce a bargaining game.

Let $T \subseteq \mathbb{R}$ denote the time space, $X \subseteq \mathbb{R}$ the set of feasible states and $A_{i} \subseteq \mathbb{R}$ the set of admissible actions for each $i \in N$. Here we consider stationary (time invariant) infinite horizon games and thus fix $T=[0, \infty)$. At each point in time $t \in T$, each agent $i \in N$ executes an action $a_{i}(t) \in A_{i}$. Let $A=\chi_{i \in N} A_{i}$ denote the jointly admissible action space. A profile of action trajectories $\boldsymbol{a}: T \rightarrow A$ determines the state trajectory $x: T \rightarrow X$ via the law of motion $f: X \times A \rightarrow X$. The transition function $f$ is a stationary differential equation

$$
\frac{\mathrm{d} x(t)}{\mathrm{d} t}=f(x(t), \boldsymbol{a}(t))
$$

which governs the evolution of the state over time and we assume that the function $f(x, \boldsymbol{a})$ is continuously differentiable in $x$ and $\boldsymbol{a}$.

Technically, we distinguish between actions and strategies. The former is an element of a set, while the latter is a function. A stationary feedback strategy is a function $\sigma_{i}: X \rightarrow A_{i}$ that determines the current action. An agent thus observes $x(t)$ and sets $a_{i}(t)$ according to $\sigma_{i}(x(t))$. Let us formally introduce the set of admissible strategies by

$$
\Sigma_{i}=\left\{\sigma_{i}: X \rightarrow A_{i} \mid \sigma_{i}(x) \text { is Lipschitz continuous on } X\right\} .
$$

The conditions imposed on the state equation $f$ and strategy spaces $\left\{\Sigma_{i}\right\}_{i \in N}$ imply that the solution $(x(t))_{t \geq 0}$ of the differential equation (1) exists and is unique as well as continuous (Başar \& Olsder, 1999, Thm. 5.1).

We further need to introduce payoffs (utility). At any position $(t, x(t)) \in$ $T \times X$ the payoff functional $u_{i}: T \times X \times \times_{i \in N} \Sigma_{i} \rightarrow \mathbb{R}$ of any agent $i \in N$ is 
given by the discounted stream of instantaneous payoffs

$$
u_{i}(t, x(t), \sigma)=\int_{t}^{\infty} e^{-r_{i}(s-t)} G_{i}(x(s), \sigma(x(s))) \mathrm{d} s .
$$

Here, $r_{i}>0$ is the time preferences rate and $G_{i}: X \times A \rightarrow \mathbb{R}$ the instantaneous utility function. The functions $\left\{G_{i}(x, \boldsymbol{a})\right\}_{i \in N}$ are assumed to be continuously differentiable in $x$ and $\boldsymbol{a}$. Since the setup is stationary the payoff functional $u_{i}$ does not depend on the current time $t$, but only on the current state $x(t)$, i.e., $u_{i}(x(t), \sigma)=u_{i}\left(t^{\prime}, x(t), \sigma\right)$ for all $t^{\prime} \in T$ and we thus suppress $t$ as an explicit argument (Caputo, 2005, Thm. 19.2). For notational convenience we denote the current state with $x=x(t)$. We denote the class of all stationary infinite horizon games by

$$
\mathcal{G}=\left\{\Gamma(x) \mid \Gamma(x)=\left\langle N,\left(\sum_{i}\right)_{i \in N},\left(u_{i}(x, \cdot)\right)_{i \in N}\right\rangle\right\} .
$$

We make two assumptions about jointly admissible strategy profiles $\sigma \in$ $\chi_{i \in N} \Sigma_{i}$. A strategy profile $\sigma$ is admissible if the state stays in the state space and the utility functional $u_{i}$ is finite for all $i$. Therefore consider the parametrized solution of (1) originating from any position $(t, x) \in T \times X$

$$
x(s)=y(s ; t, x, \sigma)=x+\int_{t}^{s} f(x(k), \sigma(x(k))) \mathrm{d} k .
$$

Now we define a set $\Sigma \subseteq \chi_{i \in N} \Sigma_{i}$ of jointly admissible strategies by

$$
\begin{gathered}
\Sigma=\left\{\sigma \in \searrow_{i \in N} \Sigma_{i} \mid \forall(t, x) \in T \times X: y(s ; t, x, \sigma) \in X \forall s \geq t,\right. \\
\left.\forall x \in X: \max _{i \in N}\left\{\left|u_{i}(x, \sigma)\right|\right\}<\infty\right\} .
\end{gathered}
$$

Given a game $\Gamma(x) \in \mathcal{G}$ as a primitive we define the set of feasible payoffs by considering all payoff allocations $\boldsymbol{u}(x, \boldsymbol{\sigma})=\left(u_{i}(x, \boldsymbol{\sigma})\right)_{i \in N}$ under admissible strategies such that $S(x) \subseteq \mathbb{R}^{n}$ is given by

$$
S(x)=\left\{\boldsymbol{u}(x, \boldsymbol{\sigma}) \in \mathbb{R}^{n} \mid \boldsymbol{\sigma} \in \Sigma\right\} .
$$

To properly define a bargaining problem we need to fix a state dependent disagreement point $\boldsymbol{d}(x) \in S(x)$. For now we assume that $\overline{\boldsymbol{\sigma}} \in \Sigma$ is a given disagreement strategy that induces for all $x \in X$ the disagreement point $\boldsymbol{d}(x)=\boldsymbol{u}(x, \overline{\boldsymbol{\sigma}})$. In Section 3 we argue that the noncooperative equilibrium payoff is a reasonable disagreement point. 
Definition 1 (Bargaining Problem). The pair $(S(x), \boldsymbol{d}(x))$ is a bargaining problem.

Usually, a bargaining solution is a point in the payoff space $S(x)$. Here, however, the agents bargain over strategies that determine the payoffs. Therefore we consider bargaining solutions as functions in the strategy space $\Sigma$. Let $\mathcal{B}=\{(S(x), \boldsymbol{d}(x)) \mid x \in X\}$ denote the set of all bargaining problems.

Definition 2 (Bargaining Solution). A bargaining solution is a correspondence $\phi: \mathcal{B} \rightrightarrows \Sigma$ that assigns to each problem $(S(x), d(x)) \in \mathcal{B}$ a set $\phi(S(x), \boldsymbol{d}(x)) \subseteq \Sigma$ of admissible strategy profiles.

We assume that the bargaining solution is the set of maximizers of a continuous function $H: \mathbb{R}^{n} \rightarrow \mathbb{R}$. The arguments of $H$ are the cooperation dividends

$$
E_{i}(x, \sigma)=u_{i}(x, \sigma)-d_{i}(x)
$$

With $\boldsymbol{E}(x, \boldsymbol{\sigma})=\left(E_{i}(x, \boldsymbol{\sigma})\right)_{i \in N}$ the bargaining solution is then given by

$$
\phi(S(x), \boldsymbol{d}(x))=\underset{\boldsymbol{\sigma} \in \Sigma}{\arg \max } H(\boldsymbol{E}(x, \boldsymbol{\sigma})) .
$$

An element of $\phi(S(x), \boldsymbol{d}(x))$ is termed a cooperative strategy and is denoted by $\boldsymbol{\sigma}_{x}^{*}$. One should note that the set $\phi(S(x), \boldsymbol{d}(x))$ may differ with respect to $x$ and a cooperative strategy thus generally depends on the current state $x$. Let us briefly illustrate the idea. Assume that the agents rely on the Nash bargaining solution to determine a cooperative strategy at $(t, x)=\left(0, x_{0}\right)$ and let us further assume that there exist two maximizers

$$
\left\{\sigma_{x_{0}}^{*, 1}, \sigma_{x_{0}}^{*, 2}\right\}=\underset{\sigma \in \Sigma}{\arg \max } \prod_{i \in N} E_{i}\left(x_{0}, \sigma\right)
$$

Now the agents reevaluate the problem at $(t, x)=\left(1, x_{1}\right) \neq\left(0, x_{0}\right)$ and end up with a unique maximizer

$$
\left\{\sigma_{x_{1}}^{*}\right\}=\underset{\sigma \in \Sigma}{\arg \max } \prod_{i \in N} E_{i}\left(x_{1}, \sigma\right) .
$$

Assume that $\sigma_{x_{0}}^{*, 1}=\sigma_{x_{1}}^{*}$ and $\sigma_{x_{0}}^{*, 2} \neq \sigma_{x_{1}}^{*}$ hold, then the first cooperative strategy $\sigma_{x_{0}}^{*, 1}$ is time consistent at $x_{1}$ and the second one $\sigma_{x_{0}}^{*, 2}$ is not. The goal of the 
article is to identify bargaining solutions on a restricted domain of games that are time consistent over the entire state space $X$ and which happen to be also individually rational.

Axiomatic bargaining theory (see e.g. Peters, 1992) studies solutions that can be uniquely identified by a number of axioms. Here we do not intend to pin down a unique bargaining solution by some suitable axiomatization, but to check whether there exists an initial cooperative strategy $\boldsymbol{\sigma}_{x_{0}}^{*} \in \phi\left(S\left(x_{0}\right), \boldsymbol{d}\left(x_{0}\right)\right)$ that $(i)$ remains individually rational throughout the game and (ii) is robust with respect to renegotiations.

We distinguish between a weak and strong notion of $(i)$ and (ii). A cooperative strategy is weak if $(i)$ and (ii) only hold along the cooperative state trajectory $x^{*}(s)=y\left(s ; 0, x_{0}, \sigma_{x_{0}}^{*}\right)$ and strong if $(i)$ and $(i i)$ hold along any admissible state trajectory $x(s)=y\left(s ; 0, x_{0}, \sigma\right), \sigma \in \Sigma$. We define the differential game concepts rigorously.

Definition 3 (Overall Individual Rationality). A solution $\phi$ satisfies Overall Individual Rationality if there exists a cooperative strategy $\boldsymbol{\sigma}_{x_{0}}^{*} \in \phi\left(S\left(x_{0}\right), \boldsymbol{d}\left(x_{0}\right)\right)$ such that

$$
\min _{i \in N}\left\{E_{i}\left(x_{0}, \sigma_{x_{0}}^{*}\right)\right\} \geq 0 .
$$

A bargaining solution satisfies OIR if each agent is not harmed by cooperating at the beginning of the game. This property is a global necessary condition for cooperation, because otherwise there would be an agent who does not want to cooperate at all. As time goes by it might be the case that cooperation under the initial agreement lacks payoff dominance with respect to the noncooperative outside option. We thus also introduce the subgame notion of individual rationality.

Definition 4 (Subgame Individual Rationality). A solution $\phi$ satisfies Weak/Strong Subgame Individual Rationality if there exists a cooperative strategy $\sigma_{x_{0}}^{*} \in$ $\phi\left(S\left(x_{0}\right), \boldsymbol{d}\left(x_{0}\right)\right)$ such that

$$
\begin{aligned}
& \min _{i \in N}\left\{E_{i}\left(x^{*}(s), \sigma_{x_{0}}^{*}\right)\right\} \geq 0 \quad \forall s \geq 0, \\
& \min _{i \in N}\left\{E_{i}\left(x, \sigma_{x_{0}}^{*}\right)\right\} \geq 0 \quad \forall x \in X .
\end{aligned}
$$

The strong version is robust in the sense that even if the cooperative path $x^{*}(s)$ is perturbed to $x(s)=x^{*}(s)+\varepsilon, \varepsilon \neq 0$, it always pays off to stick to 
the initial agreement. In order to satisfy time consistency we require that there exists an initial cooperative strategy that solves any bargaining problem occurring at a later point in time.

Definition 5 (Time Consistency). A solution $\phi$ satisfies Weak/Strong Time Consistency if

$$
\begin{aligned}
& \bigcap_{s \geq 0} \phi\left(S\left(x^{*}(s)\right), \boldsymbol{d}\left(x^{*}(s)\right)\right) \neq \emptyset, \\
& \bigcap_{x \in X} \phi(S(x), \boldsymbol{d}(x)) \neq \emptyset .
\end{aligned}
$$

Clearly, SSIR implies WSIR implies OIR and STC implies WTC. Apparently, the reverse does not hold in general.

In the literature on cooperative differential games (see e.g. Jørgensen \& Zaccour, 2002; Zaccour, 2008) there are different terminologies for the previously introduced concepts. We follow Başar (1989) in calling the renegotiation condition (ii) time consistency. It is a property that generally applies to optimal control problems as well as differential games. The strong version corresponds to subgame perfectness in standard game theory lingo and is also called Markov perfect equilibrium or feedback equilibrium. In cooperative differential games it is usually assumed that the agents jointly maximize the sum of individual payoffs in order to determine the cooperative strategies to be played throughout the game. When solving this optimal control problem via a dynamic programming approach strong time consistency is automatically given. The weak version of subgame individual rationality (WSIR) is then just called time consistency and following Kaitala \& Pohjola (1990) the strong version (SSIR) is called agreeability. Here, however, we do not assume that the agents jointly maximize their payoffs, but that they bargain over strategies. We therefore explicitly distinguish between the consistency (renegotiating) and the individual rationality condition.

\section{DISAGREEMENT POINT}

Clearly, the bargaining solution $\phi(S(x), \boldsymbol{d}(x))$ depends on the disagreement point $\boldsymbol{d}(x)=\boldsymbol{u}(x, \overline{\boldsymbol{\sigma}})$ and thus on the disagreement strategies $\overline{\boldsymbol{\sigma}} \in \Sigma$. Here the disagreement point is not given, but constructed from the underlying game $\Gamma(x)$. There is no objective rationale that yields the "correct" disagreement 
point, but we would like to argue that the Nash (1950a, 1951) equilibrium payoff is the natural choice for pure bargaining games. A Nash equilibrium is a strategy tuple such that no agent has an incentive to unilaterally change her strategy. It is straightforward to define the solution concept for differential games (Starr \& Ho, 1969a,b). Given the feedback information structure we must make sure, however, that no agent has an incentive to change her strategy (a function) for different states $x \in X$.

Definition 6 (Equilibrium). The $n$-tuple $\bar{\sigma} \in \Sigma$ is a subgame perfect Nash equilibrium for $\Gamma(x) \in \mathcal{G}$ if for all agents $i \in N$ and positions $x \in X$ the following inequalities hold:

$$
u_{i}(x, \overline{\boldsymbol{\sigma}}) \geq u_{i}\left(x, \sigma_{i}, \overline{\boldsymbol{\sigma}}_{-i}\right) \quad \forall \sigma_{i} \in \Sigma_{i}
$$

where $\overline{\boldsymbol{\sigma}}_{-i}=\left(\bar{\sigma}_{j}\right)_{j \in N \backslash\{i\}}$ are the equilibrium strategies of the opponents.

For any $x \in X$ the disagreement point $\boldsymbol{d}(x) \in \mathbb{R}^{n}$ is then given by the current payoff-to-go under the noncooperative equilibrium strategies, i.e., $d_{i}(x)=u_{i}(x, \bar{\sigma})$ for all $i \in N$.

Let us elaborate why the Nash equilibrium payoff is the natural disagreement point. Pure bargaining games are a special kind of cooperative game in the sense that either the grand coalition forms and jointly realizes the payoffs $\boldsymbol{u}\left(x, \boldsymbol{\sigma}_{x}^{*}\right) \in S(x)$ or there exists an agent who disagrees with $\boldsymbol{\sigma}_{x}^{*}$ and then every agent $i \in N$ gets $d_{i}(x)$. We therefore do not allow for the formation of any coalition $C \subset N$, but the singleton $C=\{i\}$. At the beginning of the game $(t, x)=\left(0, x_{0}\right)$ the agents can thus either jointly agree on playing $\sigma_{x_{0}}^{*} \in \Sigma$ or they cannot come to an agreement. Hence in case of disagreement the agents face a fully noncooperative game ( $n$ singletons) as of which the Nash equilibrium is the rationale solution concept with payoffs $\boldsymbol{d}\left(x_{0}\right)$. That is, they should bargain over gains compared to the outside option which is playing a noncooperative game. Let us assume the agents settled on $\sigma_{x_{0}}^{*}$ at the beginning of the game, but suppose they reopen negotiations at any position $(t, x) \in(0, \infty) \times X$. They then face the same situation as in the beginning of the game, but the outside option is rather the current Nash equilibrium payoff at position $x$ and thus $d(x)$.

By invoking dynamic programming techniques, one can show that the equilibrium strategies solve a system of coupled differential equations. The following Lemma is fundamental when characterizing the equilibrium of a differential game (see e.g. E. Dockner \& Wagener, 2014, Thm. 1). 
Lemma 1. The n-tuple $\bar{\sigma} \in \Sigma$ is a subgame perfect Nash equilibrium for $\Gamma(x) \in \mathcal{G}$ if there exist $n$ functions $\left\{d_{i}: X \rightarrow \mathbb{R}\right\}_{i \in N}$ that are continuously differentiable in $x$ and solve the following system of coupled Hamilton-JacobiBellman (HJB) equations:

$$
\begin{aligned}
r_{i} d_{i}(x) & =\max _{a_{i} \in A_{i}}\left\{G_{i}\left(x, a_{i}, \overline{\boldsymbol{\sigma}}_{-i}(x)\right)+d_{i}^{\prime}(x) f\left(x, a_{i}, \overline{\boldsymbol{\sigma}}_{-i}(x)\right)\right\} \\
& =G_{i}(x, \overline{\boldsymbol{\sigma}}(x))+d_{i}^{\prime}(x) f(x, \overline{\boldsymbol{\sigma}}(x)) .
\end{aligned}
$$

Further, the transversality condition $\lim _{t \rightarrow \infty} e^{-r_{i} t} d_{i}(x)=0$ must be satisfied for all agents $i \in N$ and all feasible states $x \in X$.

The function $d_{i}(\cdot)$ is termed value function. We apply Lemma 1 in the next section in order to establish the existence of an equilibrium in constant strategies.

\section{LINEAR-STATE GAMES}

In the present paper we restrict the domain of games under consideration to a class $\mathcal{G}_{\text {Lin }} \subset \mathcal{G}$ of linear-state games and therefore consider state equations and instantaneous payoff functions that satisfy the following functional form:

$$
\begin{aligned}
& f(x, \boldsymbol{a})=h(\boldsymbol{a})-\delta x, \\
& G_{i}(x, \boldsymbol{a})=g_{i}(\boldsymbol{a})+\eta_{i} x,
\end{aligned}
$$

where $\delta, \eta_{i} \in \mathbb{R}$ denote parameters. Finally, we assume that for all different $i, j \in N$ the state equation as well as the payoffs are independent of the actions of distinct players, i.e.,

$$
\frac{\partial^{2} h(\boldsymbol{a})}{\partial a_{j} \partial a_{i}}=0 \quad \text { and } \quad \frac{\partial^{2} g_{i}(\boldsymbol{a})}{\partial a_{j} \partial a_{i}}=0 .
$$

There is thus no multiplicative interaction between the actions of the players. Let us define the class $\mathcal{G}_{\text {Lin }}$ of linear-state games by

$$
\mathcal{G}_{\text {Lin }}=\{\Gamma(x) \in \mathcal{G} \mid \text { Eqs. (4), (5) and (6) hold }\} \text {. }
$$


Proposition 1. For $\Gamma(x) \in \mathcal{G}_{\text {Lin }}$ there exists an equilibrium in constant strategies $\overline{\boldsymbol{k}} \in A \subseteq \mathbb{R}^{n}$ with associated linear value functions $\left\{d_{i}(x)=\right.$ $\left.\alpha_{i} x+\beta_{i}\right\}_{i \in N}$. The constants $\alpha_{i}$ and $\beta_{i}$ are given by

$$
\begin{aligned}
\alpha_{i} & =\frac{\eta_{i}}{r_{i}+\delta}, \\
\beta_{i} & =\frac{1}{r_{i}}\left[g_{i}(\overline{\boldsymbol{k}})+\alpha_{i} h(\overline{\boldsymbol{k}})\right] .
\end{aligned}
$$

The equilibrium strategies $\bar{\sigma}_{i}(x)=\bar{k}_{i}$ are given by the root of the first order condition for $i \in N$

$$
L_{i}\left(\bar{k}_{i}, \alpha_{i}\right)=0
$$

where $L_{i}(\cdot, \cdot)$ denotes the derivative of the right hand side of the HJB equation with respect to the action $a_{i}$

$$
L_{i}\left(a_{i}, \alpha_{i}\right)=\frac{\partial\left[g_{i}\left(a_{i}, \overline{\boldsymbol{\sigma}}_{-i}(x)\right)+\alpha_{i} h\left(a_{i}, \overline{\boldsymbol{\sigma}}_{-i}(x)\right)\right]}{\partial a_{i}}\left(a_{i}, \alpha_{i}\right) .
$$

Proof. We guess a functional form for the value function $d_{i}(x)=\alpha_{i} x+\beta_{i}$ where $\alpha_{i}, \beta_{i} \in \mathbb{R}$ are constants. We show that $\left\{d_{i}(x)\right\}_{i \in N}$ satisfy the HJB equations of Lemma 1 . Consider the function $L_{i}(\cdot, \cdot)$ as defined by (9). Due to (6) $L_{i}(\cdot, \cdot)$ does not depend on the equilibrium strategies $\bar{\sigma}_{j}(x)$ of any other agent $j \in N \backslash\{i\}$. For all $i \in N$ let $\bar{k}_{i}$ denote the root of the first order condition (9), i.e., $\bar{k}_{i}$ solves $L_{i}\left(\bar{k}_{i}, \alpha_{i}\right)=0$. Since $\bar{k}_{i}$ only depends on $\alpha_{i}$ it is a constant. Collect the maximizers in $\bar{k}=\left(\bar{k}_{i}\right)_{i \in N}$. The maximized HJB equation (3) then reads

$$
r_{i}\left(\alpha_{i} x+\beta_{i}\right)=g_{i}(\overline{\boldsymbol{k}})+\eta_{i} x+\alpha_{i}(h(\overline{\boldsymbol{k}})-\delta x) .
$$

The maximized HJB equation is satisfied for all $x \in X$ if $\alpha_{i}$ and $\beta_{i}$ satisfy (7) and (8) respectively.

We can now state the main result. We use the information about the noncooperative equilibrium strategies and restrict the domain of admissible cooperative strategies to constants. Then we are able to show that all OIR solutions satisfy SSIR and STC. 
Proposition 2. If the space of admissible cooperative strategies is restricted to constants $\Sigma_{i}^{*}=\left\{\sigma_{i}(x)=k_{i} \mid k_{i} \in A_{i}\right\} \subset \Sigma_{i}$, then all OIR bargaining solutions satisfy STC and SSIR.

Proof. Let $\boldsymbol{k} \in A$ and for $s \geq t$ let $y(s ; t, x, \boldsymbol{k})$ denote the feasible solutions of (4) conditioned on the current position $(t, x)$

$$
y(s ; t, x, \boldsymbol{k})=\frac{1}{\delta}\left[h(\boldsymbol{k})-e^{-\delta(s-t)}(h(\boldsymbol{k})-\delta x)\right] .
$$

For any $x \in X$ the feasible current payoff-to-go is then given by

$$
\begin{aligned}
u_{i}(x, \boldsymbol{k}) & =\int_{t}^{\infty} e^{-r_{i}(s-t)}\left[g_{i}(\boldsymbol{k})+\eta_{i} y(s ; t, x, \boldsymbol{k})\right] d s \\
& =\frac{\eta_{i}}{r_{i}+\delta} x+\frac{1}{r_{i}}\left[g_{i}(\boldsymbol{k})+\frac{\eta_{i}}{r_{i}+\delta} h(\boldsymbol{k})\right] \\
& =\alpha_{i} x+\frac{1}{r_{i}}\left[g_{i}(\boldsymbol{k})+\alpha_{i} h(\boldsymbol{k})\right] .
\end{aligned}
$$

When considering constant actions $\sigma_{i}(x)=k_{i}$ for all $i \in N$, then the slope of any feasible payoff functional $\partial u_{i}(x, \boldsymbol{k}) / \partial x=\alpha_{i}$ is equal to the slope of the noncooperative equilibrium payoff $d_{i}^{\prime}(x)$. Since we fix $d_{i}(x)$ as the outside option the potential gains of cooperating from $(t, x) \in[0, \infty) \times X$ onwards become

$$
E_{i}(\boldsymbol{k})=u_{i}(x, \boldsymbol{k})-d_{i}(x)=\frac{1}{r_{i}}\left[g_{i}(\boldsymbol{k})-g_{i}(\overline{\boldsymbol{k}})+\alpha_{i}(h(\boldsymbol{k})-h(\overline{\boldsymbol{k}}))\right] .
$$

At $t=0$ an OIR solution solves

$$
\begin{array}{ll} 
& \boldsymbol{k}^{*} \in \underset{\boldsymbol{k} \in A}{\arg \max } H(\boldsymbol{E}(\boldsymbol{k})) \\
\text { s.t. } & \min _{i \in N}\left\{E_{i}(\boldsymbol{k})\right\} \geq 0 .
\end{array}
$$

As $\boldsymbol{k}^{*}$ does not depend on the state SSIR and STC are satisfied for all $x \in X$.

One should note that the result is not constructive. Restricting the set of admissible cooperative strategies to constants is not sufficient, because the bargaining solution could still vary with respect to the state. The result is 
entirely driven by the fact that the cooperation dividends $\boldsymbol{E}(\boldsymbol{k})$ do not depend on $x$. If this was not the case, i.e., the cooperation dividends do depend on the state $\boldsymbol{E}(x, \boldsymbol{k})$, then the bargaining solution $\boldsymbol{k}_{x}^{*} \in \arg \max _{\boldsymbol{k} \in A} H(\boldsymbol{E}(x, \boldsymbol{k}))$ still varies over the state space.

State invariance of the cooperative dividends $\boldsymbol{E}(\boldsymbol{k})$ has an interesting implication, because then the set of individually rational actions

$$
A^{\mathrm{IR}}=\left\{\boldsymbol{k} \in A \mid \min _{i \in N}\left\{E_{i}(\boldsymbol{k})\right\} \geq 0\right\}
$$

is also invariant with respect to $x$. The set of feasible payoffs $S(x)$ still depends on the state $x$, though. Note, however, that any bargaining problem $(S(x), \boldsymbol{d}(x))$ is only a linearly shifted variant of the initial problem

$$
(S(x), \boldsymbol{d}(x))=\left(S\left(x_{0}\right)+\boldsymbol{\alpha} \cdot\left(x-x_{0}\right), \boldsymbol{d}\left(x_{0}\right)+\boldsymbol{\alpha} \cdot\left(x-x_{0}\right)\right)
$$

where we should recall that $\boldsymbol{\alpha}=\left(\alpha_{i}\right)_{i \in N}$ are the coefficients of the value functions $d_{i}(x)=\alpha_{i} x+\beta_{i}$. Since we are only interested in OIR solutions rational agents bargain over additional surplus compared to the disagreement point - we may define a normalized problem by

$$
S^{\mathrm{IR}}=S(x)-\boldsymbol{d}(x)=\left\{\boldsymbol{E}(\boldsymbol{k}) \mid \boldsymbol{k} \in A^{\mathrm{IR}}\right\} \subseteq \mathbb{R}_{+}^{n} .
$$

As $S^{\mathrm{IR}}$ does not depend on $x$, all normalized problems coincide. Figure 1 illustrates the relationship. In Figure 1a we consider two problems $\left(S\left(x_{0}\right), \boldsymbol{d}\left(x_{0}\right)\right)$ and $\left(S\left(x_{1}\right), \boldsymbol{d}\left(x_{1}\right)\right)$ for some different states $x_{0}, x_{1} \in X$. Even though the original problems do not coincide in the payoff space $S\left(x_{0}\right) \neq S\left(x_{1}\right)$, they are equivalent in terms of current surplus $S\left(x_{0}\right)-\boldsymbol{d}\left(x_{0}\right)=S\left(x_{1}\right)-\boldsymbol{d}\left(x_{1}\right)$ (cf. Figure 1b).

There is a straightforward policy implication: if a linear-state model approximates a real world phenomenon sufficiently well and there are agents who want to jointly benefit by cooperation, then the policymaker (designer) should only allow constant strategies such that no agent has an incentive to deviate from the initial cooperative agreement as times goes by.

\section{APPLICATION}

We discuss an application drawn from environmental economics (E. J. Dockner \& Van Long, 1993). There are two countries that pollute the air. Let 


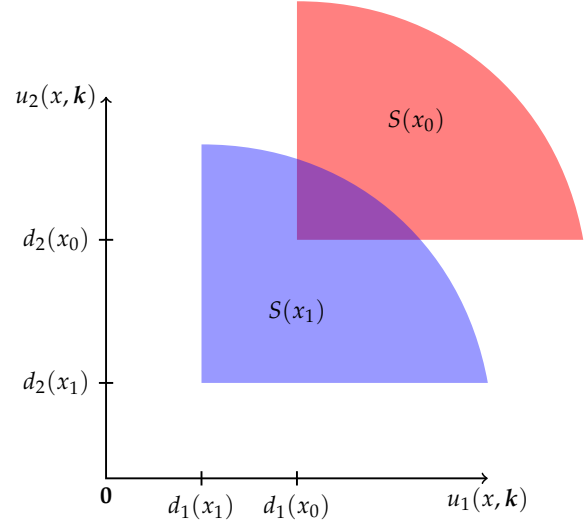

(a) original problem $(S(x), \boldsymbol{d}(x))$

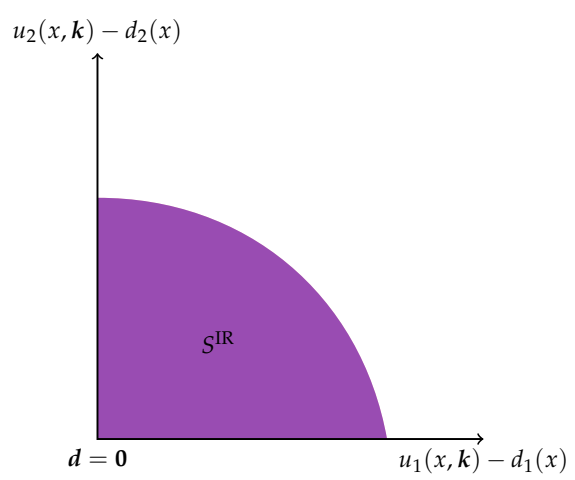

(b) normalized problem $\left(S^{\mathrm{IR}}, \mathbf{0}\right)$

Figure 1: Normalization $S^{\mathrm{IR}}=S(x)-\boldsymbol{d}(x)$

$k_{i} \in A_{i}=\mathbb{R}_{++}$denote the constant emission rate and $x \in X=\mathbb{R}_{+}$the stock of pollution. A country gets some payoff from current pollution via $g_{i}\left(k_{i}\right)=\ln \left(k_{i}\right)$, but has to take into account a stock externality described by $\eta_{i}$. As total payoffs are given by $G_{i}\left(x, k_{i}\right)=\ln \left(k_{i}\right)+\eta_{i} x$, we assume that the externality parameter is negative $\eta_{i}<0$ and payoffs thus decrease in the total stock of pollution. Let us further assume that the pollution stock increases linearly in emissions $h(\boldsymbol{k})=k_{1}+k_{2}$ and that pollution is naturally absorbed with $\delta>0$ such that the state equation reads $f(x, \boldsymbol{k})=k_{1}+k_{2}-\delta x$. The utility under constant strategies is given by (10)

$$
u_{i}\left(x, k_{i}, k_{-i}\right)=\alpha_{i} x+\frac{1}{r_{i}}\left[\ln \left(k_{i}\right)+\alpha_{i}\left(k_{i}+k_{-i}\right)\right] .
$$

It is straightforward to verify that there exists a noncooperative equilibrium characterized by the action

$$
\bar{k}_{i}=\underset{k_{i} \in A_{i}}{\arg \max }\left\{u_{i}\left(x, k_{i}, \bar{k}_{-i}\right)\right\}=-\frac{1}{\alpha_{i}} \stackrel{(7)}{=}-\frac{r_{i}+\delta}{\eta_{i}}>0 .
$$

One should verify that

$$
d_{i}(x)=u_{i}(x, \overline{\boldsymbol{k}})=\alpha_{i} x+\frac{1}{r_{i}}\left[\ln \left(-\frac{1}{\alpha_{i}}\right)-\alpha_{i}\left(\frac{1}{\alpha_{i}}+\frac{1}{\alpha_{-i}}\right)\right]
$$


in fact solves the general HJB equation

$$
r_{i} d_{i}(x)=\max _{a_{i} \in A}\left\{\ln \left(a_{i}\right)+\eta_{i} x+d_{i}^{\prime}(x)\left(a_{i}+\bar{a}_{-i}-\delta x\right)\right\} .
$$

The maximized HJB equation reads

$$
r_{i} d_{i}(x)=\ln \left(-\frac{1}{d_{i}^{\prime}(x)}\right)+\eta_{i} x-d_{i}^{\prime}(x)\left(\frac{1}{d_{i}^{\prime}(x)}+\frac{1}{d_{-i}^{\prime}(x)}+\delta x\right) .
$$

Substituting $d_{i}^{\prime}(x)=\alpha_{i}$ and dividing by $r_{i}$ yields the claimed result

$$
d_{i}(x)=\underbrace{\frac{\left(\eta_{i}-\alpha_{i} \delta\right)}{r_{i}}}_{=\alpha_{i}} x+\frac{1}{r_{i}}\left[\ln \left(-\frac{1}{\alpha_{i}}\right)-\alpha_{i}\left(\frac{1}{\alpha_{i}}+\frac{1}{\alpha_{-i}}\right)\right] .
$$

The cooperation dividend is then given by (11)

$$
E_{i}(\boldsymbol{k})=\frac{1}{r_{i}}\left[\ln \left(\frac{k_{i}}{\bar{k}_{i}}\right)-\frac{1}{\bar{k}_{i}} \sum_{j=1}^{2}\left(k_{j}-\bar{k}_{j}\right)\right]
$$

where we use $\alpha_{i}=-1 / \bar{k}_{i}$. We consider the bargaining problem

$$
(S, \mathbf{0})=\left(\left\{\left(E_{1}(\boldsymbol{k}), E_{2}(\boldsymbol{k})\right) \mid \boldsymbol{k} \in A\right\}, \mathbf{0}\right) .
$$

First, we show that there are conceptional analogies to an Edgeworth box. To be more precise: the set of individually rational cooperative actions $A^{\mathrm{IR}} \subset A$ has the shape of a lens, the noncooperative equilibrium strategies $\overline{\boldsymbol{k}}$ anchor the zero indifference curves, and the set of Pareto efficient actions - denoted by $A^{\mathrm{P}} \subset A$ - corresponds to the contract curve.

We solve $E_{i}(\boldsymbol{k})=0$ for $k_{-i}$ and denote the solution by $\hat{k}_{-i}: A_{i} \rightarrow A_{-i}$ such that $E_{i}\left(k_{i}, \hat{k}_{-i}\left(k_{i}\right)\right)=0$ for all $k_{i} \in A_{i}$. We can explicitly solve for $\hat{k}_{-i}\left(k_{i}\right)$ with

$$
\hat{k}_{-i}\left(k_{i}\right)=\bar{k}_{i} \ln \left(\frac{k_{i}}{\bar{k}_{i}}\right)-k_{i}+\sum_{j=1}^{2} \bar{k}_{j}
$$

Consider the graphs of the zero indifference curves through the point $\overline{\boldsymbol{k}}$

$$
\begin{aligned}
& \mathscr{G}\left(\hat{k}_{2}\right)=\left\{\left(k_{1}, \hat{k}_{2}\left(k_{1}\right)\right) \mid k_{1} \in A_{1}\right\} \subset A, \\
& \mathscr{G}\left(\hat{k}_{1}\right)=\left\{\left(\hat{k}_{1}\left(k_{2}\right), k_{2}\right) \mid k_{2} \in A_{2}\right\} \subset A .
\end{aligned}
$$


Now we make the following observation: the functions $\hat{k}_{-i}\left(k_{i}\right)$ are strictly concave $\hat{k}_{-i}^{\prime \prime}\left(k_{i}\right)=-\bar{k}_{i} / k_{i}^{2}<0$. The curve $\mathscr{G}\left(\hat{k}_{1}\right)$ is then strictly convex in the $A$ space. The curves are tangent $\mathscr{G}\left(\hat{k}_{2}\right) \cap \mathscr{G}\left(\hat{k}_{1}\right)=\{\overline{\boldsymbol{k}}\}$ for the trivial problem $A^{\mathrm{IR}}=\{\overline{\boldsymbol{k}}\}$, but they must intersect twice $\left|\mathscr{G}\left(\hat{k}_{2}\right) \cap \mathscr{G}\left(\hat{k}_{1}\right)\right|=2$ for a nontrivial problem $\left|A^{\mathrm{IR}}\right|>1$ and thus describe a lens.

We can further construct a curve of Pareto efficient action pairs (contract curve) and determine the resulting Pareto frontier $\partial S \subset S$. Define the set of efficient actions by

$$
A^{\mathrm{P}}=\left\{\begin{array}{l|l|l}
\boldsymbol{k} \in A & \frac{\partial E_{1}(\boldsymbol{k}) / \partial k_{1}}{\partial E_{1}(\boldsymbol{k}) / \partial k_{2}}=\frac{\partial E_{2}(\boldsymbol{k}) / \partial k_{1}}{\partial E_{2}(\boldsymbol{k}) / \partial k_{2}}
\end{array}\right\} .
$$

The contract curve $\tilde{k}_{2}:\left(0, \bar{k}_{1}\right) \rightarrow\left(0, \bar{k}_{2}\right)$ given by

$$
\tilde{k}_{2}\left(k_{1}\right)=-\frac{\bar{k}_{2}}{\bar{k}_{1}} k_{1}+\bar{k}_{2}
$$

solves

$$
\frac{\partial E_{1}\left(k_{1}, \tilde{k}_{2}\left(k_{1}\right)\right) / \partial k_{1}}{\partial E_{1}\left(k_{1}, \tilde{k}_{2}\left(k_{1}\right)\right) / \partial k_{2}}=\frac{\partial E_{2}\left(k_{1}, \tilde{k}_{2}\left(k_{1}\right)\right) / \partial k_{1}}{\partial E_{2}\left(k_{1}, \tilde{k}_{2}\left(k_{1}\right)\right) / \partial k_{2}} .
$$

The efficient actions are then given by $A^{\mathrm{P}}=\left\{\left(k_{1}, \tilde{k}_{2}\left(k_{1}\right)\right) \mid k_{1} \in\left(0, \bar{k}_{1}\right)\right\}$. There is a relationship between the contract curve and the Pareto frontier $\partial S$ in the sense that each point on the contract curve defines a point on the Pareto frontier such that

$$
\partial S=\left\{\left(E_{1}(\boldsymbol{k}), E_{2}(\boldsymbol{k})\right) \mid \boldsymbol{k} \in A^{\mathrm{P}}\right\} .
$$

From an economic point of view there are two axioms that should be shared by all bargaining solutions, namely: individual rationality as well as group rationality (efficiency). We thus pick a solution from the set $A^{\mathrm{IR}, \mathrm{P}}=A^{\mathrm{IR}} \cap A^{\mathrm{P}}$.

Let us illustrate the results with a numerical example. Consider the following parametrization $\left(r_{1}, r_{2}, \eta_{1}, \eta_{2}, \delta\right)=(0.10,0.15,-1,-1,1)$. The equilibrium actions are directly given by $\overline{\boldsymbol{k}}=(1.10,1.15)$. We are going to solve for the Egalitarian $\boldsymbol{k}^{E}$ and Utilitarian solution $\boldsymbol{k}^{U}$ respectively defined by

$$
\begin{aligned}
& \boldsymbol{k}^{E}=\underset{\boldsymbol{k} \in A^{\mathrm{IR}}}{\arg \max }\left\{\min \left\{E_{1}(\boldsymbol{k}), E_{2}(\boldsymbol{k})\right\}\right\}, \\
& \boldsymbol{k}^{U}=\underset{\boldsymbol{k} \in A^{\mathrm{IR}}}{\arg \max }\left\{E_{1}(\boldsymbol{k})+E_{2}(\boldsymbol{k})\right\} .
\end{aligned}
$$


The two solutions are polar cases describing full egalitarianism

$$
E_{1}\left(\boldsymbol{k}^{E}\right)=E_{2}\left(\boldsymbol{k}^{E}\right)
$$

and maximal efficiency in the sense of surplus maximization

$$
E_{1}\left(\boldsymbol{k}^{U}\right)+E_{2}\left(\boldsymbol{k}^{U}\right) \geq E_{1}(\boldsymbol{k})+E_{2}(\boldsymbol{k}) \quad \forall \boldsymbol{k} \in A^{\mathrm{IR}} .
$$

Since both solutions are located on the contract curve $\tilde{k}_{2}\left(k_{1}\right)$, it is straightforward to calculate them. The action $k_{1}^{E}=0.5047$ solves $E_{1}\left(k_{1}^{E}, \tilde{k}_{2}\left(k_{1}^{E}\right)\right)=$ $E_{2}\left(k_{1}^{E}, \tilde{k}_{2}\left(k_{1}^{E}\right)\right)$ such that the Egalitarian solution is given by $\left.\boldsymbol{k}^{E}=\left(k_{1}^{E}, \tilde{k}_{2}\left(k_{1}^{E}\right)\right)\right)=$ $(0.5047,0.6224)$. For the Utilitarian solution one simply solves

$$
k_{1}^{U}=0.6717=\underset{k_{1}>0}{\arg \max }\left\{E_{1}\left(k_{1}, \tilde{k}_{2}\left(k_{1}\right)\right)+E_{2}\left(k_{1}, \tilde{k}_{2}\left(k_{1}\right)\right)\right\} .
$$

The Utilitarian solution is then given by $\left.\boldsymbol{k}^{U}=\left(k_{1}^{U}, \tilde{k}_{2}\left(k_{1}^{U}\right)\right)\right)=(0.6717,0.4478)$.

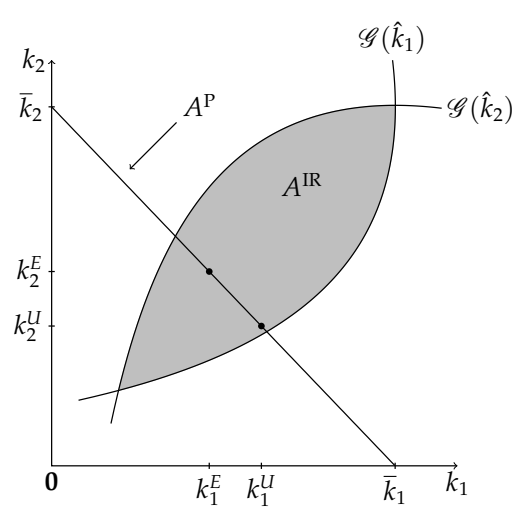

(a) action space

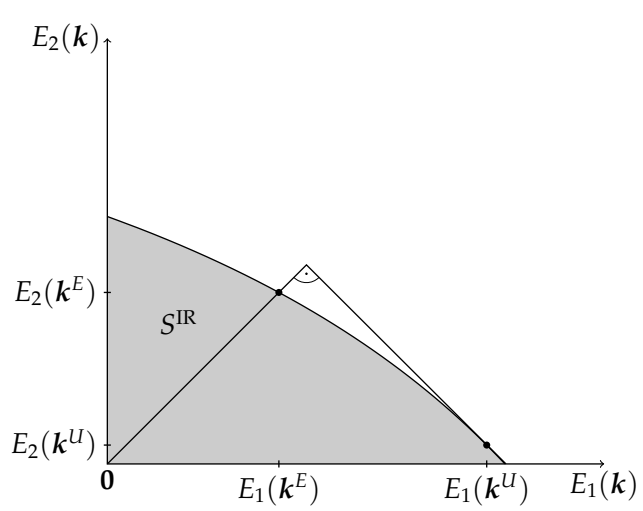

(b) payoff space

Figure 2: Egalitarian and Utilitarian bargaining solutions

Figure 2 illustrates the solutions in the action space $A$ (cf. Figure $2 \mathrm{a}$ ) and IR payoff space $S^{\mathrm{IR}}$ (cf. Figure $2 \mathrm{~b}$ ). We find that the emission rate of country 1 is less under egalitarianism than under utilitarianism $k_{1}^{E}<k_{1}^{U}$ and vice versa for country 2. Apparently the results are driven by the heterogeneous discount rates $r_{1}<r_{2}$, because country 1 can be considered more patient than country 2 . 
Since country 2 discounts future payoffs more heavily than country 1, it would be also able to emit more pollutants in order to equalize the surplus.

On the other hand, if we were only concerned about total surplus maximization, then country 1 is favored, because it does not discount future payoffs that heavily. In Figure $2 b$ we find the geometric analogon of the analytic results. The Egalitarian solution is given by the intersection of the diagonal and the Pareto frontier, while the Utilitarian solution is given by the tangential point of the line with slope -1 and the Pareto frontier.

\section{CONCLUSION}

We addressed the question of identifying subgame individually rational (SIR) and time consistent (TC) bargaining solutions for a variety of linear-state differential games. For these kind of games there exists a subgame perfect equilibrium in constant strategies. We showed that all overall individually rational bargaining solutions satisfy SIR and TC, if one restricts the space of admissible cooperative strategies to constants. The approach of using cooperative strategies that are functionally equivalent to given equilibrium strategies should be applicable more broadly to design SIR and TC cooperative solutions. In subsequent work we hope to generalize the results for the rich class of linear-quadratic games. There seems to be an inverse relationship, however, between the complexity of the model and the bargaining solutions that satisfy SIR and TC. In an accompanying paper (Hoof, 2020) we showed for a nonlinear model of dynamic cake eating that a bargaining solution must maximize some linear-homogenous function in order to satisfy SIR and TC. Generally, we plan to pin down classes of games such that bargaining solutions can be uniquely identified by SIR and/or TC and that those two properties thus serve as axioms in dynamic models.

\section{References}

Başar, T. (1989). Time Consistency and Robustness of Equilibria in Non-Cooperative Dynamic Games. In F. van der Ploeg \& A. de Zeeuw (Eds.), Dynamic policy games in economics (Vol. 181, pp. 9 - 54). Elsevier.

Başar, T., \& Olsder, G. J. (1999). Dynamic Noncooperative Game Theory (2nd ed.). SIAM. 
Caputo, M. R. (2005). Foundations of Dynamic Economic Analysis. Cambridge University Press.

Dockner, E., \& Wagener, F. (2014). Markov perfect Nash equilibria in models with a single capital stock. Economic Theory, 56(3), 585 - 625.

Dockner, E. J., Jørgensen, S., Long, N. V., \& Sorger, G. (2000). Differential Games in Economics and Management Science. Cambridge University Press.

Dockner, E. J., \& Van Long, N. (1993). International Pollution Control: Cooperative versus Noncooperative Strategies. Journal of Environmental Economics and Management, 25(1), 13 - 29.

Haurie, A. (1976). A note on nonzero-sum differential games with bargaining solution. Journal of Optimization Theory and Applications, 18(1), 31 - 39.

Hoof, S. (2020). A pure bargaining game of dynamic cake eating. (mimeo)

Jørgensen, S., Martín-Herrán, G., \& Zaccour, G. (2003). Agreeability and Time Consistency in Linear-State Differential Games. Journal of Optimization Theory and Applications, 119(1), $49-63$.

Jørgensen, S., \& Zaccour, G. (2002). Time Consistency in Cooperative Differential Games. In G. Zaccour (Ed.), Decision \& control in management science (pp. 349 366). Springer.

Kaitala, V., \& Pohjola, M. (1990). Economic Development and Agreeable Redistribution in Capitalism: Efficient Game Equilibria in a Two-Class Neoclassical Growth Model. International Economic Review, 31(2), 421 - 438.

Liu, P. T. (1973). Nonzero-sum differential games with bargaining solutions. Journal of Optimization Theory and Applications, 11(3), 284 - 292.

Nash, J. F., Jr. (1950a). Equilibrium points in $n$-person games. Proceedings of the National Academy of Sciences, 36(1), 48 - 49.

Nash, J. F., Jr. (1950b). The Bargaining Problem. Econometrica, 18(2).

Nash, J. F., Jr. (1951). Non-Cooperative Games. Annals of Mathematics, 54(2), 286 295.

Peters, H. J. (1992). Axiomatic Bargaining Game Theory (Vol. 9). Springer.

Petrosyan, L. A., \& Zaccour, G. (2018). Cooperative Differential Games with Transferable Payoffs. In T. Basar \& G. Zaccour (Eds.), Handbook of dynamic game theory (pp. 595 -632). Springer.

Starr, A. W., \& Ho, Y. C. (1969a). Further Properties of Nonzero-Sum Differential Games. Journal of Optimization Theory and Applications, 3(4), 207 - 219.

Starr, A. W., \& Ho, Y. C. (1969b). Non-Zero Sum Differential Games. Journal of Optimization Theory and Applications, 3(3), 184 - 206.

Yeung, D. W. K., \& Petrosyan, L. A. (2018). Nontransferable Utility Cooperative Dynamic Games. In T. Başar \& G. Zaccour (Eds.), Handbook of dynamic game theory (pp. 633 -670). Springer. 
Zaccour, G. (2008). Time Consistency in Cooperative Differential Games: A Tutorial. INFOR: Information Systems and Operational Research, 46(1), 81 - 92. 